\title{
Trombose arterial em microcirculação pós-Covid 19: Relato de caso
}

\author{
Arterial trombosis in microcirculation after Covid 19: Case report \\ Trombosis arterial en microcirculación post-Covid 19: Informe de caso
}

Recebido: 13/03/2021 | Revisado: 20/03/2021 | Aceito: 10/04/2021 | Publicado: 20/04/2021

\author{
João Victor Carvalho Barbosa \\ (ORCID: https://orcid.org/0000-0001-7790-0312 \\ Universidade Federal do Delta do Parnaíba, Brasil \\ E-mail: joaovictorcarvalhob@gmail.com \\ Priscylla Frazão Rodrigues \\ ORCID: https://orcid.org/0000-0001-7580-516X \\ Universidade Federal do Delta do Parnaíba, Brasil \\ E-mail: priscyllafrazao1995@gmail.com \\ Cláudio Vinícius Barroso Queirós de Lima \\ ORCID: https://orcid.org/0000-0002-4047-8637 \\ Universidade Federal do Delta do Parnaíba, Brasil \\ E-mail: cld.vinicius99@gmail.com \\ Olívio Joaquim Fonseca Neto \\ ORCID: https://orcid.org/0000-0002-2695-0077 \\ Universidade Federal do Delta do Parnaíba, Brasil \\ E-mail: oliviofonsecaneto@ hotmail.com \\ Isabella Pires Gomes Mendes \\ ORCID: https://orcid.org/0000-0003-3486-7908 \\ Universidade Federal do Delta do Parnaíba, Brasil \\ E-mail: isabellapiresmendes@gmail.com \\ Andressa Carvalho Pereira \\ ORCID: https://orcid.org/0000-0001-7721-0661 \\ Universidade Federal do Delta do Parnaíba, Brasil \\ E-mail: andressa_carvalho@ufpi.edu.br \\ Brenda Larissa Andrade Viana \\ ORCID: https://orcid.org/0000-0003-2285-4309 \\ Universidade Federal do Delta do Parnaíba, Brasil \\ E-mail: brendalarissa.av@gmail.com \\ Elias de Carvalho Magalhães Neto \\ ORCID: https://orcid.org/0000-0002-7494-7147 \\ Universidade Federal do Delta do Parnaíba, Brasil \\ E-mail: elias.nt@hotmail.com
}

\begin{abstract}
Resumo
O presente artigo tem como objetivo relatar o caso de um paciente do sexo masculino, com diagnóstico de COVID19, e posterior apresentação de trombose arterial em microcirculação, assim como divulgar o manejo do caso. Paciente, 48 anos, do sexo masculino, foi internado com quadro de Síndrome Respiratória Aguda Grave (SRAG) após diagnóstico de COVID-19, havendo comprometimento de $60 \%$ da capacidade funcional dos pulmões. Após melhora do quadro respiratório, o paciente passou a apresentar dor súbita na mão esquerda, cianose não fixa, perfusão lentificada e hiperemia reativa, porém, ainda apresentando pulso ulnar e pulso radial palpáveis. Após o exame físico, foram solicitados novo exame de testagem de COVID-19, hemograma, leucograma, proteína C reativa, dímero D, tempo de protrombina e tempo de tromboplastina parcial ativado. A partir dos resultados de todos os exames foi fechado o diagnóstico de trombose da microcirculação arterial decorrente de infecção por SARS-CoV-2. A conduta adotada teve como objetivo tratar os problemas de circulação do paciente. Até o quarto dia após início do tratamento o paciente se apresentava sem queixas de dor, com melhoras na perfusão na região afetada, com a mão aquecida e com discretas áreas de necrose na região das polpas digitais.
\end{abstract}

Palavras-chave: Trombose; COVID-19; Síndrome Respiratória Aguda Grave.

\begin{abstract}
This article aims to report the case of a male patient, diagnosed with COVID-19, and subsequent presentation of microcirculatory arterial thrombosis, as well as to publicize the management of the case. A 48-year-old male patient was admitted with Severe Acute Respiratory Syndrome (SARS) after diagnosis of COVID-19, with impairment of $60 \%$ of the lungs functional capacity. After improvement of the respiratory condition, the patient started to present sudden pain in the left hand, unfixed cyanosis, slow perfusion and reactive hyperemia, however, still had palpable ulnar and radial pulse. After the physical examination, a new test for COVID-19, hemogram, leukogram, C-reactive protein, D-dimer, prothrombin time and activated partial thromboplastin time were requested. From the results of all exams, the diagnosis of thrombosis in arterial microcirculation as a result of the infection by SARS-CoV-2 was
\end{abstract}


confirmed. One approach adopted was to treat the patient's circulation problems. Up to the fourth day after the start of treatment, the patient presented no complaints of pain, with improvements in the perfusion in the affected region, with a warm hand and discrete areas of necrosis in the region of the digital pulps.

Keywords: Thrombosis; COVID-19; Severe Acute Respiratory Syndrome.

\section{Resumen}

Este artículo tiene como objetivo reportar el caso de un paciente masculino, diagnosticado de COVID-19, y posterior presentación de trombosis arterial microcirculatoria, así como dar a conocer el manejo del caso. Un paciente masculino de 48 años ingresó con Síndrome Respiratorio Agudo Severo (SARS) después del diagnóstico de COVID19, con deterioro del $60 \%$ de la capacidad funcional pulmonar. Luego de la mejoría de la condición respiratoria, el paciente comenzó a presentar dolor brusco en la mano izquierda, cianosis no fijada, perfusión lenta e hiperemia reactiva, sin embargo, aún tenía pulso cubital y radial palpables. Tras la exploración física se solicitó una nueva prueba de COVID-19, hemograma, leucograma, proteína C reactiva, dímero $\mathrm{D}$, tiempo de protrombina y tiempo de tromboplastina parcial activada. A partir de los resultados de todos los exámenes, se confirmó el diagnóstico de trombosis en la microcirculación arterial como resultado de la infección por SARS-CoV-2. Un enfoque adoptado fue el de tratar los problemas circulatorios del paciente. Hasta el cuarto día después del inicio del tratamiento, la paciente no presentó quejas de dolor, con mejoría de la perfusión en la región afectada, con mano cálida y discretas áreas de necrosis en la región de las pulpas digitales.

Palabras clave: Trombosis; COVID-19; Síndrome Respiratorio Agudo Grave.

\section{Introduçãa}

A doença causada pelo vírus SARS-CoV-2 tem sido responsável por altas taxas de internação em Unidades de Terapia Intensiva (UTIs) e alta mortalidade. Além da insuficiência respiratória, a coagulopatia vem se tornando uma complicação recorrente em pacientes com COVID-19, especialmente os mais graves (Llitjos et al., 2020). Isso ocorre devido o SARS-CoV2 induzir uma tempestade de citocinas que, em última análise, leva à ativação da cascata de coagulação, causando eventos trombóticos (Dolhnikoff et al., 2020). Ainda, interações medicamentosas entre terapias para COVID-19 e agentes antiplaquetários e anticoagulantes podem, também, contribuir para o estado pró-trombótico encontrado nesta doença (Passos et al., 2020). Estados de hipercoagulabilidade e alterações hematológicas já foram descritas, sendo a elevação do Dímero-D um importante marcador de desfechos desfavoráveis (Ramos \& Ota-Arakaki, 2020)

Alguns estudos já fizeram a associação entre a infecção por SARS-CoV-2 e o risco de fenômenos tromboembólicos. Uma série retrospectiva com 183 pacientes diagnosticados com COVID-19 relatou que 71,4\% dos não sobreviventes preencheram os critérios de coagulação intravascular disseminada (DIC), enquanto apenas $0,6 \%$ dos sobreviventes o fizeram (Helms et al., 2020). Outro estudo retrospectivo francês encontrou $69 \%$ de tromboembolismo venoso (TEV) em pacientes com COVID-19 anticoagulados em UTI, após o uso de uma triagem sistemática (Llitjos et al., 2020). Ademais, dados de necropsias de pacientes com COVID-19 revelaram trombose na microcirculação, apoiando a teoria atual do estado de hipercoagulabilidade (Dolhnikoff et al., 2020).

A anticoagulação profilática em pacientes com COVID-19 pode ser recomendada (Llitjos et al., 2020). Tang et al. (2020) sugeriram que o uso de heparina por 7 dias ou mais resultou em diminuição da mortalidade em casos graves. No entanto, mais pesquisas são necessárias para que um consenso seja estabelecido na literatura.

\section{Metodologia}

O presente artigo trata-se de um relato de caso, cuja abordagem é descritiva e qualitativa, baseada no método descrito por (Pereira et al., 2018). O presente relato visa expor o manejo de paciente com trombose arterial em microcirculação pós quadro de COVID-19. Para isso, foi feita a análise dos prontuários e exames complementares solicitados ao paciente para análise e construção do relato. O termo de consentimento livre esclarecido (TCLE) foi devidamente assinado pelo paciente, o qual reconheceu o trabalho e permitiu a publicação do caso. 


\section{Relato de caso}

O presente caso clínico foi selecionado entre os pacientes atendidos no Centro Integrado de Especialidades MédicasCIEM (fomentado pela FAPEPI). Homem, 48 anos, internado no dia 18 de janeiro de 2021, com quadro de Síndrome Respiratória Aguda Grave (SRAG), após confirmação diagnóstica de COVID-19 por amostras de swab de nasofaringe no dia 14 de janeiro de 2021 (Figura 1). Paciente queixou-se de desconforto respiratório iniciado a cerca de dois dias e demandava oxigenoterapia. Ao exame de tomografia computadorizada (TC) dos pulmões, eles apresentaram comprometimento de $60 \%$ da sua capacidade funcional. Fez uso de ceftriaxona por sete dias, de azitromicina por cinco dias, de oseltamivir por cinco dias e de dexametasona por sete dias. Paciente recebeu alta no dia 25 de janeiro de 2021 sem queixas ou sintomas respiratórios, com saturação O2 = 92-93\% em ar ambiente, após $48 \mathrm{~h}$ sem oxigenoterapia. Foi, então, encaminhado para acompanhamento na Unidade Básica de Saúde.

$\mathrm{Na}$ avaliação ambulatorial, dia 26 de janeiro de 2021, paciente queixou-se de dor intensa na mão esquerda, sendo, pois, encaminhado para consulta com cirurgião vascular. Durante a consulta, realizada no dia 28 de janeiro de 2021, paciente relatou dor súbita na mão há cerca de 2 dias. Ao realizar exame físico, observou-se que a mão esquerda apresentava cianose não fixa, perfusão lentificada e hiperemia reativa (Figura 2). Ainda assim, as artérias radial e ulnar possuíam, ambas, pulso palpável. Foi solicitado novo exame de testagem para COVID-19, hemograma, leucograma, proteína C reativa, dímero D, tempo de protrombina, tempo de tromboplastina parcial ativado e um eco-doppler arterial de membro superior esquerdo.

Figura 1 - Laudo do teste rápido de COVID-19

confirmando o diagnóstico.

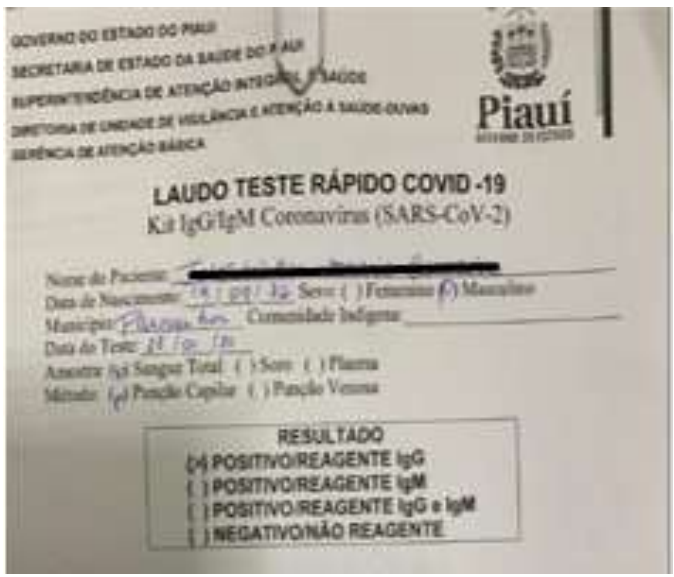

Fonte: Arquivo pessoal dos autores.
Figura 2 - Cianose não fixa, perfusão lentificada e hiperemia reativa.

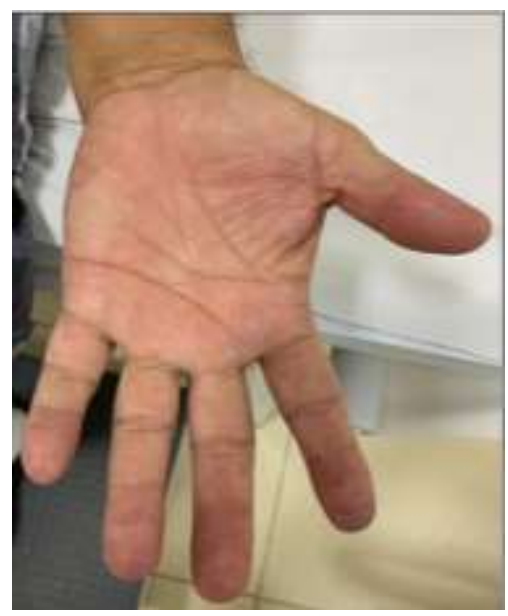

Fonte: Arquivo pessoal dos autores.

O hemograma revelou que a quantidade de hemácias estava um pouco abaixo dos valores de referência, estando em 4,46 milhões por milímetro cúbico (valor de referência mínimo era de 4,5 milhões por milímetro cúbico). Já a contagem de leucócitos indicava uma leucopenia, por haver 4160 por milímetro cúbico e a margem mínima do valor de referência ser de 5000. Além disso, a porcentagem do hematócrito também estava abaixo da preconizada, em 38,6\% (valor de referência mínimo é de 40\%). O resultado do exame de detecção da proteína C reativa deu negativo e o da quantidade de dímero D estava dentro dos valores de referência. O tempo de protrombina e o tempo de tromboplastina parcial ativado também se situavam dentro dos limites preconizados. O resultado do exame detector de COVID-19 foi positivo/reagente IgG. Por fim, o ecodoppler indicou a ausência de alterações hemodinâmicas nas artérias subclávia, axilar, braquial, radial e ulnar. A partir disso, foi fechado o diagnóstico de trombose da microcirculação arterial decorrente de infecção por SARS-CoV-2. 
A conduta adotada a partir disso objetivou incidir sobre os problemas de circulação do paciente, com o uso de Rivaroxabana 15 miligramas a cada 12 horas e de Cilostazol 100 miligramas também a cada 12 horas, e sobre a dor neuropática, com Thioctacid 600 miligramas a cada 24 horas, Pregabalina 75 miligramas a cada 12 horas e Lisador 500 miligramas a cada 6 horas. Ademais, o paciente foi orientado a reter calor na região da mão por meio do uso de uma luva, a não submeter a região a esforços físicos e a adotar uma postura mais cuidadosa de modo a evitar traumas locais.

Até o quarto dia após o início do tratamento, o paciente se apresentava sem queixas de dor, com melhoras na perfusão na região afetada, com a mão aquecida e com discretas áreas de necrose na região das polpas digitais.

\section{Discussão}

O SARS-CoV-2, relatado pela primeira vez em Wuhan, China, é causador da pandemia do COVID-19, estando relacionado à SRAG, além de outras complicações locais e sistêmicas, como alterações cardiovasculares e metabólicas (Roberto, Pacheco, Gusmão \& Gabriel, 2020). Outra possível consequência da infecção é a associação com o desenvolvimento de fenômenos trombóticos: sugere-se que ocorra devido a ligação do vírus - por sua alta afinidade - com a enzima conversora de angiotensina 2 (ECA-2) e o estímulo à sua “downregulation”, inibindo a transformação da angiotensina I (Agt I). A Agt I, acumulada, provocaria um estado de inflamação pelo organismo e, como resultado, hipercoagulabilidade e coagulação intravascular disseminada (Avila, Long, Holladay \& Gottlieb, 2021).

No que diz respeito à sintomatologia respiratória, o paciente necessitou de suplementação de oxigênio devido a dispneia referida determinada pela SRAG e, após isso, foi realizada a TC de tórax. Conforme Rosa et al. (2020), a TC se constitui como uma ótima ferramenta diagnóstica para os casos de COVID-19 em virtude de suas altas sensibilidade e especificidade, auxiliando na busca por complicações, na definição da extensão e do grau de acometimento pulmonar e, claro, para confirmação de suspeita, em especial no contexto de casos atípicos da doença. Constatou-se que $60 \%$ da capacidade funcional pulmonar já estava comprometida, nesse caso. Além disso, o paciente apresentou as manifestações vasculares após a infecção, observadas no exame físico e analisadas por exames laboratoriais, corroborando ainda mais para a hipótese que relaciona a infecção por coronavírus e os fenômenos tromboembólicos.

Em casos de trombose arterial, observam-se sinais e sintomas relacionados à hipoperfusão tecidual e isquemia da área, a depender do tempo de oclusão do vaso, da sua localização e da existência ou não de circulação colateral (Bortoluzzi, Oliveira, Fraga, Santos \& Piantá, 2017). Ainda de acordo com Bortoluzzi et al. (2017), a dor intensa e progressiva, em especial em regiões distais, caracteriza-se como o sintoma mais comum desta patologia - o paciente do caso a apresentou na mão esquerda. Da mesma forma, reforça sobre a alteração do tempo de enchimento capilar, que se torna lento ou ausente, o qual foi avaliado e se encontrava lentificado. Em se tratando da hiperemia reativa, a qual foi analisada e confirmada no caso, é diretamente proporcional ao grau de isquemia e, portanto, é um teste que pode ser utilizado para confirmar se há ou não fluxo sanguíneo inadequado para a área afetada (Wolosker, Pereira \& Frankini, 2005). Embora, também segundo Bortoluzzi et al. (2017), sejam prevalentes outros sinais e sintomas como parestesia, ausência de pulso, palidez, paralisia e hipotermia, o paciente não os manifestou.

Ainda que não totalmente estabelecida, a investigação de complicações trombóticas em pacientes com COVID-19, segundo sugere a literatura, deve conter exames laboratoriais como hemograma, D-dímero, Coagulograma, com atenção especial para o Tempo de Protrombina (TP), Proteína C Reativa (PCR), entre outros (BRANDÃO et al., 2020; Oliveira Junior \& Lourenço, 2020). No caso relatado, foram realizados os seguintes exames: novo exame de testagem para COVID-19, hemograma, PCR, D-dímero, TP e Tempo de Tromboplastina Parcial Ativado (TTPA).

Quantos aos resultados, estudos demonstram que alterações laboratoriais relacionadas com a coagulação frequentes em pacientes com COVID-19, e que podem ser preditoras de evolução complicada, são o aumento da síntese de trombina e 
diminuição da fibrinólise, além de valores elevados de D-dímero, aumento do fibrinogênio, tempo prolongado de protrombina, trombocitopenia, linfopenia, neutrofilia, leucopenia (Brandão, Silva, Ramos, Melo \& Sarinho, 2020); Barros, Maia, Marques, Prette-Junior, Fiorelli \& Cerqueira, 2020; Fleury, 2020). O paciente do caso, no entanto, não apresentou a maioria dessas alterações, possuindo resultado negativo da PCR, assim como D-dímero, TP e TTPA dentro dos valores de referência.

As alterações significativas no caso foram no hemograma, o qual mostrou alterações como leucopenia, já estabelecida como possibilidade nesses casos, além de alterações no eritrograma, representadas pela diminuição tanto na quantidade de hemácias quanto da porcentagem do hematócrito.

Ainda sobre a investigação clínica, Bortoluzzi et al. (2017) traz que alguns exames complementares como exame com Doppler e a Angiotomografia computadorizada (angioTC) podem ser ferramentas úteis para auxiliar no diagnóstico de trombose arterial ao contribuir para a avaliação do fluxo arterial e para a determinação do local e da natureza da oclusão, respectivamente. A angio TC poderia, então, também ter sido utilizada para auxiliar no caso relatado.

Segundo França et al. (2017), os estudos envolvendo o manejo de isquemias no membro superior de origem trombótica são escassos. Entretanto, ainda segundo os mesmos autores, apesar de não ser bem estabelecida e ainda necessitar de mais estudos, a conduta conservadora envolvendo os anticoagulantes orais tem sido usada e se mostrou eficaz quanto ao efeito antitrombótico (França et al., 2017). No caso relatado, foi prescrito o medicamento Xarelto (Rivaroxabana), um novo anticoagulante oral. Também fez parte do tratamento do paciente o Vasogard (Cilostazol), que tem sido o medicamento de escolha para tratar pacientes com claudicação (França et al., 2017; Portaria SES-DF No 1045, 2019; Mota, Santos, Silva, Mesquita \& Oliveira, 2017).

Além disso, com relação à dor neuropática, o paciente utilizou os seguintes remédios: Thioctacid (ácido tiótico), Pregabalina e Lisador. De acordo com González-Escalada (2005), a Pregabalina é um fármaco de efeito analgésico utilizado no tratamento de dor neuropática, enquanto que, segundo os estudos de Lee et al. (2005), o uso do medicamento Thioctacid mostrou benefícios para a função endotelial e vascular.

\section{Conclusão}

Em suma, os dados apresentados no caso corroboram para a associação do SARS-CoV-2 com um alto potencial para causar trombose da microcirculação arterial em pacientes, que podem se relacionar com o estado de hipercoagulabilidade e coagulação intravascular disseminada provocada pelo vírus. O reconhecimento de complicações de forma precoce e o suporte otimizado é uma importante estratégia terapêutica. No caso da ocorrência de trombose, alguns sinais e sintomas podem se apresentar, como dor no local e sinais de isquemia, o reconhecimento precoce possui alto valor para conduta clínica. Além disso, o acompanhamento dos fatores de coagulação em pacientes com Covid-19 através de exames laboratoriais como hemograma, D-dímero, Coagulograma, Tempo de Protrombina (TP) se mostra um fator importante na identificação de complicações precoces, favorecendo o prognóstico dos pacientes.

O manejo clínico ideal na SARS-CoV-2 ainda não está bem estabelecido, porém é importante a observação dos sinais clínicos e intervenção sobre as principais complicações secundárias à infecção. A coleta de história clínica detalhada auxilia na identificação de pacientes suscetíveis a alterações vasculares e permite a prevenção de agravos. Dessa forma, a aplicação dos protocolos existentes e o tratamento baseado em evidências científicas, além das terapias que englobam as principais complicações, perfazem uma conduta adequada

Sugere-se, ainda, que mais pesquisas sejam feitas, tratando do COVID-19 e suas complicações, a fim de entender como a fisiopatologia da doença altera as funções fisiológicas do organismo humano e nos predispõe a essas complicações, como o alto potencial de causar trombose. Assim, condutas mais firmes e eficazes surgirão no manejo e tratamento do paciente infectado pelo SARS-CoV-2. 


\section{Referências}

Avila, J., Long, B., Holladay, D., \& Gottlieb, M. (2021). Thrombotic complications of COVID-19. The American Journal of Emergency Medicine, 39, 213218. https://doi.org/10.1016/j.ajem.2020.09.065

Barros, B. C. S., Maia, A. B., Marques, M. A., Prette-Junior, P. R., Fiorelli, S. K. A., Cerqueira, F. D. C., Barros, B. C. S., Maia, A. B., Marques, M. A., Prette-Junior, P. R., Fiorelli, S. K. A., \& Cerqueira, F. D. C. (2020). A atuação da Angiologia e da Cirurgia Vascular na pandemia de COVID-19. Revista do Colégio Brasileiro de Cirurgiões, 47. https://doi.org/10.1590/0100-6991e-20202595

Bortoluzzi, B. N., Oliveira, E. A., Fraga, M. B., Santos, M. P. H. dos. \& Piantá, R. M. (2017). Oclusão arterial aguda. Biblioteca Virtual de Saúde, Acta méd., Porto Alegre, 38 .

Brandão, S. C. S., Silva, E. T. A. G. B. de B. e, Ramos, J. de O. X., Melo, L. M. M. P. de, \& Sarinho, E. S. C. (2020). Covid-19, Imunidade, Endotélio E Coagulação: Compreenda A Interação Mariola Comunicação. https://repositorio.ufpe.br/handle/123456789/37570

Dolhnikoff, M., Duarte-Neto, A. N., Monteiro, R. A. de A., Silva, L. F. F. da, Oliveira, E. P. de, Saldiva, P. H. N., Mauad, T., \& Negri, E. M. (2020). Pathological evidence of pulmonary thrombotic phenomena in severe COVID-19. Journal of Thrombosis and Haemostasis, 18(6), 1517-1519. https://doi.org/10.1111/jth.14844

Fleury, M. K. (2020). A COVID-19 e o laboratório de hematologia: uma revisão da literatura recente. Revista Brasileira de Análises Clínicas, $52(2), 131-7$.

França, B. A., Monteiro, A. M., Carvalho, R. de S., Vasconcelos, J. C., Silva, S. A. da, \& Júnior, A. F. da C. (2017). Isquemia de membro superior: Um desafio diagnóstico e terapêutico - relato de caso. Revista de Patologia do Tocantins, 4(4), 16-21. https://doi.org/10.20873/uft.2446-6492.2017v4n4p16

González-Escalada, J. R. (2005). Pregabalina en el tratamiento del dolor neuropático periférico. Revista de la Sociedad Española del Dolor, 12(3), 169-180.

Helms, J., Tacquard, C., Severac, F., Leonard-Lorant, I., Ohana, M., Delabranche, X., Merdji, H., Clere-Jehl, R., Schenck, M., Fagot Gandet, F., Fafi-Kremer, S., Castelain, V., Schneider, F., Grunebaum, L., Anglés-Cano, E., Sattler, L., Mertes, P.-M., \& Meziani, F. (2020). High risk of thrombosis in patients with severe SARS-CoV-2 infection: A multicenter prospective cohort study. Intensive Care Medicine, 46(6), 1089-1098. https://doi.org/10.1007/s00134-02006062-X

Lee, S. R., Jeong, M. H., Lim, S. Y., Hong, S. N., Kim, K. H., Sohn, I. S., Hong, Y. J., Park, H. W., Kim, J. H., Kim, W., Ahn, Y. K., Cho, J. G., Park, J. C., \& Kang, J. C. (2006). The Effect of Alpha Lipoic Acid (Thioctacid HR®) on Endothelial Function in Diabetic and Hypertensive Patients. Korean Circulation Journal, 36(8), 559. https://doi.org/10.4070/kcj.2006.36.8.559

Llitjos, J.-F., Leclerc, M., Chochois, C., Monsallier, J.-M., Ramakers, M., Auvray, M., \& Merouani, K. (2020). High incidence of venous thromboembolic events in anticoagulated severe COVID-19 patients. Journal of Thrombosis and Haemostasis, 18(7), 1743-1746. https://doi.org/10.1111/jth.14869

Mota, T. D. C., Santos, J. D. M., Silva, B. D. J. C. D., Mesquita, N. M. C. B. D., \& Oliveira, D. M. (2017). Doença Arterial Obstrutiva Periférica: Revisão Integrativa. Revista Uningá, 53(1), Article 1. http://revista.uninga.br/index.php/uninga/article/view/1402

Oliveira Junior, R. B. de. \& Lourenço, P. M. (2020). Alterações laboratoriais e a COVID-19. Revista Brasileira de Análises Clínicas, 52(2), pp. 198-200.

Passos, H. D., Alves, M. C., Baumworcel, L., Vieira, J. P. C., Garcez, J. D. S., Sousa, A. C. S., Passos, H. D., Alves, M. C., Baumworcel, L., Vieira, J. P. C., Garcez, J. D. S., \& Sousa, A. C. S. (2020). Infecção pelo SARS-Cov-2 e Tromboembolismo Pulmonar - Comportamento Pró-Trombótico da COVID-19. Arquivos Brasileiros de Cardiologia, 115(1), 142-145. https://doi.org/10.36660/abc.20200427

Pereira, A. S., Shitsuka, D. M., Parreira, F. J., \& Shitsuka, R. (2018). Metodologia da pesquisa científica. UFSM.

Portaria SES-DF Nº 1045 de 20 de dezembro de 2019. Dispõe sobre Organização da Assistência ao Portador de Doença Arterial Obstrutiva Periférica. Diário Oficial do Distrito Federal. http://webcache.googleusercontent.com/search?q=cache:-HOnNec9S4wJ:www.saude.df.gov.br/wpconteudo/uploads/2018/04/Protocolo-Doen\%25C3\%25A7a-Arterial-Obstrutiva-Perif\%25C3\%25A9rica.pdf $+\& \mathrm{~cd}=1 \& \mathrm{hl}=\mathrm{pt}-\mathrm{BR} \& \mathrm{ct}=\mathrm{clnk} \& \mathrm{gl}=\mathrm{br}>$.

Ramos, R. P., Ota-Arakaki, J. S., Ramos, R. P., \& Ota-Arakaki, J. S. (2020). Thrombosis and anticoagulation in COVID-19. Jornal Brasileiro de Pneumologia, 46(4). https://doi.org/10.36416/1806-3756/e20200317

Roberto, G. A., Pacheco, L. R., Gusmão, M. R., \& Gabriel, S. A. (2020). COVID-19 e eventos tromboembólicos. 10.

Rosa, M. E. E., Matos, M. J. R. de, Furtado, R. S. O. de P., Brito, V. M., Amaral, L. T. W., Beraldo, G. L., Fonseca, E. K. U. N., Chate, R. C., Passos, R. B. D., Teles, G. B. da S., Silva, M. M. A., Yokoo, P., Yanata, E., Shoji, H., Szarf, G., Funari, M. B. de G., Rosa, M. E. E., Matos, M. J. R. de, Furtado, R. S. O. de P., \& Funari, M. B. de G. (2020). Achados da COVID-19 identificados na tomografia computadorizada de tórax: Ensaio pictórico. Einstein (São Paulo), 18. https://doi.org/10.31744/einstein_journal/2020rw5741

Tang, N., Bai, H., Chen, X., Gong, J., Li, D., \& Sun, Z. (2020). Anticoagulant treatment is associated with decreased mortality in severe coronavirus disease 2019 patients with coagulopathy. Journal of Thrombosis and Haemostasis, 18(5), 1094-1099. https://doi.org/10.1111/jth.14817

Wolosker, N., Pereira, A. H. \& Frankini, A. D. (2005). Diagnóstico da Doença Arterial Obstrutiva Periférica. Jornal Vascular Brasileiro, 4(3), 4. 